\title{
Philosophiques
}

\section{Narbonne, Jean-Marc, Hénologie, ontologie et Ereignis (Plotin -Proclus Heidegger), Paris, Les Belles Lettres (" L'âne d'or »), 2001, 377 p.}

\section{Daniel Mazilu}

Volume 29, numéro 1, printemps 2002

Spinoza sous le prisme de son anthropologie

URI : https://id.erudit.org/iderudit/009575ar

DOI : https://doi.org/10.7202/009575ar

Aller au sommaire du numéro

Éditeur(s)

Société de philosophie du Québec

ISSN

0316-2923 (imprimé)

1492-1391 (numérique)

Découvrir la revue

Citer ce compte rendu

Mazilu, D. (2002). Compte rendu de [Narbonne, Jean-Marc, Hénologie, ontologie et Ereignis (Plotin -Proclus Heidegger), Paris, Les Belles Lettres ( L'âne d'or »), 2001, 377 p.] Philosophiques, 29(1), 161-163. https://doi.org/10.7202/009575ar d'utilisation que vous pouvez consulter en ligne.

https://apropos.erudit.org/fr/usagers/politique-dutilisation/ 
enfin libre. Mais elle doit le demeurer. Aussi est-il nécessaire de condamner ceux qui savent tirer profit d'un régime politique, quel qu'il soit, et parviennent à le dénaturer. Les fils de Brutus représentent précisément ceux qui, par l'instigation d'un régime et de ses lois, savent immédiatement où trouver les avantages des inégalités inévitables engendrées par les lois nouvelles. Pour préserver l'isonomie, l'égalité de tous devant la loi, Brutus doit aussi punir ses fils. Au cours des chapitres suivants, Vatter explique à nouveaux frais le concept d'isonomie en montrant que cette égalité devant la loi est tributaire du mouvement historique du " retour aux origines ». Suivant Vatter, un aspect important du lien étroit entre philosophie de l'histoire et philosophie politique chez Machiavel est l'idée suivant laquelle le chemin historique des États les ramène à leurs origines, non pas à leurs formes premières, mais avant même qu'ils ne prennent une forme constitutionnelle. Ce processus suppose d'abord que l'on puisse s'interroger sur ce qui, dans la forme de l'État, a permis à certains de vivre mieux que les autres citoyens. Ce même processus suppose en second lieu que l'État soit modifié afin de prévenir les inégalités perçues comme des résultats du précédent régime constitutionnel. Mais ces deux moments ne sont eux-mêmes possibles que si on accepte la nécessité d'une suspension du rapport entre gouvernants, chefs de l'État, et gouvernés, ce que Vatter nomme la «transgression de la loi ».

Il reste beaucoup à dire sur cette étude. Mais l'essentiel est de voir à quel point la lecture radicale que propose Vatter du concept de liberté chez Machiavel pose problème pour quiconque voudrait inscrire cet auteur dans la lignée républicaine. Plusieurs commentateurs ont déjà, par le passé, exposé leurs objections à la lecture républicaine de Machiavel. Songeons à Léo Strauss et ses disciples, H. Mansfield par exemple. Mais à ma connaissance, Vatter est le seul à avoir tenté de faire une critique " interne » de la lecture républicaine de Machiavel, c'est-à-dire en fonction des propres arguments de cette tradition. Vatter ne nie pas dans son principe l'interprétation républicaine de Machiavel, mais il tente, avec beaucoup de finesse, de faire comprendre quelles seraient les conclusions véritables d'un tel modèle d'interprétation. Par sa facture complexe, le livre de Vatter en découragera plus d'un. Il s'agit toutefois, à mon avis, d'une des interprétations les plus stimulantes qu'il soit possible de lire à l'heure actuelle sur Machiavel. En outre, Vatter a le grand mérite de connaître parfaitement l'érudition française et italienne sur Machiavel, en plus, évidemment, des travaux du monde anglo-saxon. Ce travail minutieux occupera, à mon avis, une place importante pour les études machiavéliennes et l'histoire des idées, mais également pour sa contribution originale et féconde à l'élucidation des énigmes que nous adresse encore le concept de liberté politique.

CHRISTIAN NADEAU

Université de Montréal

\section{Narbonne, Jean-Marc, Hénologie, ontologie et Ereignis (Plotin - Proclus - Heidegger), Paris, Les Belles Lettres (« L'âne d'or »), 2001, 377 p.}

Fruit d'une recherche assidue menée pendant quatre années (1997-2000) et subventionnée par la Fondation allemande Humboldt et le CRSH, le dernier livre de JeanMarc Narbonne relève le défi redoutable d'un dialogue concerté de trois figures majeures de la métaphysique occidentale : Plotin, Proclus et Heidegger. Le concert 
est, à vrai dire, plutôt dissonant étant donné que Heidegger se trouve en faux, selon Narbonne, par la perspective partiale et partielle qu'il offre de la métaphysique occidentale. On s'attendrait, en lisant la trilogie conceptuelle du titre, à tomber sur la clé susceptible d'ouvrir une voie d'accès non seulement à la plus obscure notion heideggerienne, l'Ereignis (signifiant ordinairement, c'est-à-dire dans un sens non-obscurci par Heidegger, événement ou avènement), mais, par elle, au dilemme du rapport de l'ontologie à l'hénologie. Le propos de Narbonne est cependant tout autre. Son intention est double : d'abord, et c'est l'un des mérites du travail, elle consiste à retracer l'histoire de deux traditions parallèles, l'hénologie issue du platonisme et l'ontologie d'allégeance aristotélicienne ; ensuite, c'est de montrer en quoi Heidegger a finalement manqué son ambition déclarée de couvrir l'ensemble de la métaphysique occidentale - pour, éventuellement, mieux la contester par après et en même temps s'imposer lui-même comme l'unique représentant de l'authentique sens de l'être. Mais que peut-il encore rester de la pertinence d'une déploration de l'oubli de l'être quand cet être, avant même d'avoir été posé comme tel et reconnu dans son autonomie par un principe de loin supérieur qui le surplombe et le conditionne même dans sa totalité, est dépassé ? Quelle valeur pouvons-nous encore accorder donc à partir de là à l'invocation heideggerienne du retour à la pensée de l'être alors qu'il semble ignorer cette école néoplatonicienne qui place l'essentiel justement au-dessus de l'être, non pour nier ce dernier dans ses prérogatives plus ou moins légitimes à la prolifération protéiforme qui le caractérise, mais pour mieux le relativiser en fonction d'une exigence qui se réclame en ligne directe de Parménide, le héraut même de la métaphysique de Heidegger, et davantage encore de Platon, comme un défi presque ?

Après un parcours de longue haleine à travers tous les détours de la doctrine néoplatonicienne, avec un passage en revue du rôle architectonique et transcendant de l'Un, de son privilège par rapport au multiple et à l'être, c'est Heidegger qui est pris à partie pour n'avoir pas su voir ce qui, par son absence de l'ordre des déterminations sensibles, visibles, palpables, paraît néant alors qu'il est tout, s'affirme en se retirant, se manifeste en restant impénétrable, l'un au-delà de l'être. Il est à regretter seulement que l'oubli de l'être de Heidegger n'ait pas été mis en parallèle avec l'oubli de Dieu évoqué par Plotin dans l'Ennéade V, 1, 1, et qu'il ne soit pas davantage question tout le long du livre de l' " âme oublieuse ", perdue dans la contemplation, que l'on trouve en IV, 3, 32. Tout aussi regrettable, compte tenu du titre du livre, nous semble l'omission d'un passage (VI, 8, 11.35-37) où Plotin affirme qu'en somme il n'advient jamais rien, qu'il n'y a donc, en réalité, pas d'événement (Ereignis) - selon la version correcte de H.D. Saffrey ("La Théosophie de Tübingen », Recherches sur le néoplatonisme après Plotin, Vrin, 1990, p. 22-23). Cette position néoplatonicienne aurait pu être exploitée avec beaucoup de profit en vue d'une confrontation féconde entre la position heideggerienne dominée par le souci du temps et de l'histoire et la finalité néoplatonicienne de l'accès à l'éternité et au salut de l'âme.

L'ouvrage de Narbonne comporte deux parties dont la première trace une histoire et donne un exposé détaillé du néoplatonisme et dont la seconde confronte Heidegger à ses propres assertions en illustrant les points névralgiques de sa conception de l'onto-théologie. Une abondante moisson de textes néoplatoniciens augmente l'intérêt de l'ensemble : de longs extraits de Plotin (les Ennéades, bien sûr), mais aussi de Proclus, notamment d'une œuvre peu utilisée et néanmoins capitale pour la compréhension de l'École d'Athènes, la Théologie platonicienne. Le livre est aussi muni d'un abondant apparat de notes placé à la fin du livre et de trois index fort utiles : des 
passages cités, des noms anciens et un troisième de noms modernes de la philosophie et de l'érudition. La seule lacune technique à signaler est l'absence d'une bibliographie des textes utilisés.

Rappelons que la mise en plein jour du problème de la prééminence de l'ontologie ou de l'hénologie l'une sur l'autre remonte à la parution, en Allemagne, du livre d'Albert Zimmermann, Ontologie oder Metaphysik (E.J. Brill, Leiden-Köln, 1965) qui ouvrait la discussion amorcée aux XIII ${ }^{\mathrm{e}}$ et $\mathrm{XIV}^{\mathrm{e}}$ siècles sur l'objet véritable de la métaphysique. Il fut suivi de près par celui de Hans Joachim Krämer, Der Ursprung der Geistmetaphysik (B.R. Grüner, Amsterdam, 1967). Nous renvoyons, pour la clarification de l'Ereignis chez Heidegger, à l'article de Philippe Verstraeten paru dans Les Études philosophiques ( ${ }^{\circ}$ 66, janvier-mars 1986, p. 113-133) : "Le sens de l'Ereignis dans Temps et être ".

DANIEL MAZILU

Université de Montréal

\section{Brezis, David, Kierkegaard et les figures de la paternité, Paris, Cerf, La « nuit surveillée », 1999, 389 pages.}

Chargé de recherche au CNRS, David Brezis nous offre ici un travail d'une grande qualité. Dans la foulée de ses travaux précédents (Temps et présence. Essai sur la conceptualité kierkegaardienne, Paris, Vrin 1989), Brezis poursuit son étude de Kierkegaard en réinterprétant les catégories philosophiques à la lumière tamisée de la biographie de Kierkegaard. Le projet de l'A. est énoncé au début de l'ouvrage : "Réinscrivant l'œuvre kierkegaardienne dans les strictes limites de son espace textuel, nous cherchons moins à en dégager l'unité fondamentale qu'à la relire à partir de tout ce qui la met fondamentalement en contradiction avec elle-même. La soumettant à une interprétation strictement immanente, nous proposons un Kierkegaard par lui-même qui est aussi, à chaque instant, un Kierkegaard contre lui-même " (p. 10). Il s'agira d'éviter l'erreur de lire Kierkegaard en "pur » philosophe ou en "pur » biographe : on voudra plutôt analyser sa pensée dans son rapport aux figures paternelles à travers leurs " traces textuelles ", c'est-à-dire de mettre son existence en correspondance avec sa conceptualité.

L'A. explorera la pensée de Kierkegaard en plaçant au centre de son étude la figure du père, figure décisive de l'œuvre s'il en est une, tout en cherchant à " mettre en question " le logos unique assurant l'unité de tout le discours philosophique (p. 15). Il le fera en débordant la figure du père vers d'autres figures auxiliaires, comme celle du frère de Søren, Peter, l'évêque Mynster, Hegel et Dieu. Cela dit, voyons plus exactement comment l'ouvrage est construit et de quelle manière il entend cerner la pensée de Kierkegaard dans ses rapports multiples et équivoques au père.

Les deux premiers chapitres traitent de points biographiques bien connus de l'œuvre, à savoir le "Tremblement de terre » et le drame des fiançailles (pp. 17-104). On y apprend que Kierkegaard fait très tôt une découverte qui influencera sa pensée : la défaillance du père. Cette découverte de la faiblesse de l'autorité, que Kierkegaard interprète comme une tromperie, se mesure à la manière dont il se positionne face à d'autres figures de l'autorité : l'évêque Mynster, Hegel et Dieu. La conséquence est décisive pour l'œuvre : le père, comme Mynster, prêchent d'agir en conformité avec la 